\title{
Comparison of phenotypic and molecular tests to identify lactic acid bacteria
}

\author{
Paula Mendonça Moraes, Luana Martins Perin, \\ Abelardo Silva Júnior, Luís Augusto Nero \\ Departamento de Veterinária, Universidade Federal de Viçosa, Viçosa, MG, Brazil.
}

Submitted: May 14, 2011; Returned to authors for corrections: March 1, 2012; Approved: July 2, 2012.

\begin{abstract}
Twenty-nine lactic acid bacteria (LAB) isolates were submitted for identification using Biolog, API50CHL, 16S rDNA sequencing, and species-specific PCR reactions. The identification results were compared, and it was concluded that a polyphasic approach is necessary for proper LAB identification, being the molecular analyzes the most reliable.
\end{abstract}

Key words: lactic acid bacteria, identification, 16sDNA, PCR, Biolog, API.

Lactic acid bacteria (LAB) are Gram-positive, nonsporulating, microaerophilic bacteria that produce lactic acid as the main fermentation product, and they occur naturally in several food systems. Numerous studies have shown that these autochthonous microorganisms improve technological and sensory properties in food systems, and that they also help to inhibit the development of spoilage and pathogenic microorganisms (Riley and Wertz, 2002; Chen and Hoover, 2003; Deegan et al., 2006). Nowadays, consumers demand natural products, with fewer chemical preservatives, so a new approach could be the controlled use of LAB as biopreservatives. Studies are continually being conducted to identify and characterize new LAB with this usage potential (Deegan et al., 2006).

Because many LAB have similar nutritional and growth requirements, biochemical-based methodologies for identification are not conclusive in many cases. The conventional microbiological methods for bacterial identification are based on morphological and physiological characteristics such as Gram staining, cell shape, spore formation, enzyme production and the fermentation of different carbohydrates. Considering these methods, the API system (BioMerieux, Marcy l'Etoile, France) and Biolog (Biolog, Hayward, CA) are both widely used. Both methodologies are based on the fermentation patterns presented by the microorganisms. The API strips used for LAB identification are 50CHL, as the results are given as a combination of the fermentation of 49 carbohydrates and esculin hydrolysis. Biolog is a unique plate used for Gram-positive and Gram-negative bacteria and it analyses the fermentation of 96 carbohydrates. The results of both tests were analysed using computer software programs, ApiWeb and MicroLog 3, respectively. Currently, several molecular-based methodologies are available and are being implemented to identify microorganisms. One of the most useful methods is $16 \mathrm{~S}$ rDNA gene sequencing. The genome of all bacteria contains this conserved gene, and the small variability in this region is unique and specific to each species (Mohania et al., 2008).

Considering the available techniques for identifying $\mathrm{LAB}$, the objective of this study was to compare molecular and phenotypic methods for identifying $\mathrm{LAB}$ isolates: API50CHL, Biolog, 16S rDNA sequencing, and speciesspecific PCR reactions.

Twenty-nine microbial isolates were randomly selected from a LAB culture collection isolated from raw milk and cheese, that presented antimicrobial activity against Listeria monocytogenes and Staphylococcus aureus (Ortolani et al., 2010). All isolates were grown on deMan Rogosa Sharpe agar (MRS, Oxoid Ltd., Basingstoke, UK) supplemented with $25 \%$ glycerol and kept at $-80{ }^{\circ} \mathrm{C}$. An aliquot of $200 \mu \mathrm{L}$ of the stock was transferred to tubes containing $5 \mathrm{~mL}$ of MRS broth (Oxoid) and incubated at $35^{\circ} \mathrm{C}$ for $24 \mathrm{~h}$. These isolates were submitted for distinct tests (phenotypic and molecular) for identification at the genera and species level.

The obtained cultures were submitted for phenotypic identification using the Biolog system. Aliquots of the cul- 
tures were transferred to GP2 plate wells and incubated at $37^{\circ} \mathrm{C}$ for $24-48 \mathrm{~h}$, when positive results were recorded according to colour changes. The results obtained were automatically read and analysed using MicroLog 3 software, which provided the most probable genera and species of the tested culture. As phenotypic analysis, the obtained cultures were also submitted to identification using the API system; aliquots of the cultures were transferred to a CHL broth (BioMérieux), homogenized and transferred to API galleries according to the manufacturer's recommendations. After $48 \mathrm{~h}$ incubation, positive results were recorded according to colour changes. Then, the set of results was analysed using APIWeb software, which provided the most probable genera and species of the tested culture.

For molecular approach, the obtained cultures were recovered in MRS broth and aliquots of $1 \mathrm{~mL}$ were transferred to microcentrifuge tubes and centrifuged at 14,000 $\mathrm{g}$ for $2 \mathrm{~min}$, and the cell pellet was submitted to DNA extraction using the DNA Purification Kit - Wizard Genomic (ref A11125, Promega Corporation, Madison, WI). 16S rDNA sequencing was conducted according Sterr et al. (2009), and PCR products were sequenced by Macrogen Inc. (Seoul, Korea), using the five primers described by Sterr et al. (2009). The results obtained were compared with the previously deposited sequences in the National Center of Biotechnology Information database (NCBI, http:/www.ncbi.nlm.nih.gov) with the Basic Alignment Search Tool (BLAST, http://www.ncbi.nlm.nih.gov/blast).

Considering the genus results obtained by the sequencing analysis, some isolates were additionally submitted for species-specific PCR reactions to perform a complete identification of Enterococcus, Lactococcus and Lactobacillus at species level, according to primers previously described by Dutka-Malen et al. (1995), Beimhfor et al. (1997) and Nishitani et al. (2004). The amplification products were mixed with GelRed 20x (Biotium Inc., Hay- ward, CA, USA) at 5:1 proportions and submitted to electrophoresis in $1 \%$ agarose gel in TBE $0.5 \mathrm{x}$ and visualized under UV light. The results obtained from the four methodologies were compared in order to verify coinciding results, using the results of molecular identification by $16 \mathrm{~S}$ rDNA as reference. Only the results with reliability higher than $98 \%$ were considered as final results.

The 16S rDNA sequencing of all of the microorganisms identified them at the genera level, at least. Considering that species-specific PCR protocols target specific genes of genera and species, the reliability of these tests was considered to be $100 \%$. The phenotypic tests did not present such high reliable rates; for the BioLog results, the reliability ranged from 74 to $99.9 \%$, and for API50CHL the reliability ranged from 78.2 to $99.9 \%$. Even with high reliability rates, these two phenotypic tests did not coincide with the molecular reference tests for the majority of the tested isolates: when the identification results by Biolog were compared to $16 \mathrm{~S}$ rDNA gene sequencing and species-specific PCR reactions, 15 (51.7\%) and 25 (86.2\%) isolates presented divergent results, respectively; considering the results obtained by API 50CHL, 25 (86.2\%) and 26 $(89.7 \%)$ isolates presented divergent results when compared to $16 \mathrm{~S}$ rDNA sequencing and species-specific PCR, respectively.

The different methods of evaluation provided different patterns of genera and species identification for the LAB isolates (Table 1). Regarding the results by sequencing of $16 \mathrm{~S}$ rDNA gene and species-specific PCR, the most frequent genera observed was Enterococcus spp., whereas API50CHL mostly identified Lactobacillus spp., and BioLog Enterococcus spp. (Table 1 and 2). When the two phenotypic methods were compared to the sequencing results, the high frequency of non-coincident results is evident (Tables 2). However, the species-specific PCR reactions confirmed the $16 \mathrm{~S}$ rDNA results of 16 out of the

Table 1 - Frequencies of each genera and species of lactic acid bacteria identified using four different methods: 16s DNA sequencing, species-specific PCR, API50CH and BioLog.

\begin{tabular}{lcccc}
\hline Genera/specie & BioLog & API 50CH & 16s rDNA sequencing & Species-specific PCR \\
\hline Enterococcus spp. & 0 & 0 & 20 & 0 \\
Enterococcus faecalis & 5 & 0 & 0 & 9 \\
Enterococcus durans & 4 & 0 & 0 & 0 \\
other species & 6 & 0 & 0 & 0 \\
Lactococcus spp. & 0 & 0 & 0 & 0 \\
Lactococcus lactis & 1 & 6 & 3 & 2 \\
Lactobacillus spp. & 0 & 1 & 0 & 0 \\
Lactobacillus plantarum & 0 & 14 & 5 & 5 \\
Lactobacillus paracasei & 0 & 6 & 0 & 0 \\
Streptococcus spp. & 2 & 0 & 1 & 0 \\
Other genera/specie & 6 & 1 & 0 & 0 \\
Not identified & 5 & 1 & 0 & 13 \\
\hline
\end{tabular}


29 tested isolates $(55.2 \%)$, indicating their reliability for LAB identification (Table 1 and 2). However, the remaining 13 isolates $(44.8 \%)$ were not identified by the species-specific PCR reactions considered in the present study, indicating the necessity of a wider variety of primers to identify other specific genes related to $\mathrm{LAB}$ species and subspecies, or even the sequencing of other regions of $\mathrm{LAB}$ genome (Velasco et al., 2004; Mohania et al., 2008). Differences between sequencing and phenotypic tests have already been observed previously, not just for LAB but also for many other bacteria (Aymerich et al., 2003; Velasco et al., 2004; Gomes et al., 2008).

Despite being the most reliable test, sequencing has some disadvantages. Because it is so conserved, in some cases the 16S rDNA gene is not sufficient for differentiating between species of LAB, especially Enterococcus spp. (Aymerich et al., 2003; Mohania et al., 2008). Considering the difficulties in differentiating between some LAB species with $16 \mathrm{~S}$ rDNA sequencing and phenotypic tests $(6$, 12), the application of specific molecular techniques such as species-specific PCR is necessary. For example, the

Table 2 - Comparison of lactic acid bacteria identification results obtained with 16S rDNA sequencing (above 98\% reliability), species-specific PCR (100\% reliability), API50CH and BioLog.

\begin{tabular}{|c|c|c|c|c|}
\hline & BioLog & API50CH & 16S rDNA sequencing & Species-specific PCR \\
\hline LAB 01 & Enterococcus faecalis & Lactobacillus spp. & Enterococcus spp. & Enterococcus faecalis \\
\hline LAB 02 & NI & Lactobacillus plantarum & Enterococcus spp. & Enterococcus faecalis \\
\hline LAB 03 & NI & $\begin{array}{l}\text { Lactobacillus paracasei subsp } \\
\text { paracasei }\end{array}$ & Enterococcus spp. & Enterococcus faecalis \\
\hline LAB 04 & Enterococcus durans & $\begin{array}{l}\text { Lactobacillus paracasei subsp } \\
\text { paracasei }\end{array}$ & Enterococcus spp. & Enterococcus faecalis \\
\hline LAB 05 & Enterococcus faecalis & Lactobacillus plantarum & Enterococcus spp. & Enterococcus faecalis \\
\hline LAB 06 & Enterococcus faecalis & Pediococcus spp & Enterococcus spp. & NI \\
\hline LAB 07 & Enterococcus faecium & Lactobacillus plantarum & Enterococcus spp. & Enterococcus faecalis \\
\hline LAB 08 & Pediococcus acidilactci/parvulus & Lactococcus lactis subsp lactis & Enterococcus spp. & NI \\
\hline LAB 09 & Streptococcus uberis & Lactococcus lactis subsp lactis & Enterococcus spp. & NI \\
\hline LAB 10 & Pediococcus spp & Lactococcus lactis subsp lactis & Enterococcus spp. & NI \\
\hline LAB 11 & Enterococcus spp & Lactobacillus plantarum & Enterococcus spp. & NI \\
\hline LAB 12 & Enterococcus faecalis & $\begin{array}{l}\text { Lactobacillus paracasei } \\
\text { paracasei }\end{array}$ & Enterococcus spp. & Enterococcus faecalis \\
\hline LAB 13 & Enterococcus hirae & $\begin{array}{l}\text { Lactobacillus paracasei } \\
\text { paracasei }\end{array}$ & Enterococcus spp. & Enterococcus faecalis \\
\hline LAB 14 & Enterococcus durans & Lactobacillus plantarum & Enterococcus spp. & NI \\
\hline LAB 15 & Enterococcus durans & Lactobacillus plantarum & Enterococcus spp. & NI \\
\hline LAB 16 & Enterococcus durans & Lactobacillus plantarum & Enterococcus spp. & NI \\
\hline LAB 17 & Enterococcus gallinarum & Lactobacillus plantarum & Enterococcus spp. & NI \\
\hline LAB 18 & Enterococcus gallinarum & Lactobacillus plantarum & Enterococcus spp. & NI \\
\hline LAB 19 & Tetragenococcus halophilus & Lactobacillus plantarum & Enterococcus spp. & NI \\
\hline LAB 20 & Enterococcus faecalis & Lactobacillus plantarum & Enterococcus spp. & Enterococcus faecalis \\
\hline LAB 21 & NI & Lactobacillus plantarum & Lactobacillus plantarum & Lactobacillus plantarum \\
\hline LAB 22 & Pediococcus pentosaceus & $\begin{array}{l}\text { Lactobacillus paracasei } \\
\text { paracasei }\end{array}$ & Lactobacillus plantarum & Lactobacillus plantarum \\
\hline LAB 23 & Pediococcus spp & $\begin{array}{l}\text { Lactobacillus paracasei } \\
\text { paracasei }\end{array}$ & Lactobacillus plantarum & Lactobacillus plantarum \\
\hline LAB 24 & NI & Lactobacillus plantarum & Lactobacillus plantarum & Lactobacillus plantarum \\
\hline LAB 25 & Pediococcus urinaeequi & Lactococcus lactis subsp lactis & Lactobacillus plantarum & Lactobacillus plantarum \\
\hline LAB 26 & Streptococcus hyovaginalis & Lactococcus lactis subsp lactis & Lactococcus lactis subsp lactis & NI \\
\hline LAB 27 & Enterococcus gallinarum & Lactococcus lactis subsp lactis & Lactococcus lactis subsp lactis & Lactococcus lactis subsp lactis \\
\hline LAB 28 & NI & NI & Lactococcus lactis & Lactococcus lactis subsp lactis \\
\hline LAB 29 & Lactococcus lactis subsp lactis & Lactobacillus plantarum & Streptococcus spp. & NI \\
\hline
\end{tabular}

NI: Not identified. 
identification of Enterococcus spp. is based on the detection of the d-alanine-ligase enzyme, antibiotic resistance and specific regions (Dutka-Malen et al., 1995). Speciesspecific PCR is also useful for identifying microorganisms at subspecies level, which cannot be easily achieved with other common techniques (Beimfohr et al., 1997). Another disadvantage is the time required to obtain the sequencing results, which is considerably higher than the time needed to obtain phenotypic test results.

The phenotypic tests are useful for identifying a small number of isolates, especially clinical samples, as their software databases mainly contain clinically important bacteria. However, when using these methods their limitations must be considered, such as their poor reproducibility, a result of the plasticity of bacterial growth, the logistic difficulties for large-scale applications, and their sometimes poor discriminatory power (Mohania et al., 2008). Another issue is that bacterial isolates do not express all their genes at the same time, as gene expression is related to environmental conditions. Considering this, the identification of such isolates only by biochemical tests can be jeopardized.

In conclusion, the choice of identification method must be carefully analysed. Some factors must be considered, such as the origin of the samples (food or clinical isolates), the number of isolates to be identified, and staff qualifications. For clinical samples, a phenotypic test could be used as a trial test, but the molecular methods are more accurate and should be used as confirmatory tests for hardto-identify isolates. Considering that most of the LAB isolation and identification studies aim to detect potential biopreservative isolates from food, the molecular approach is probably the most sensitive.

\section{Acknowledgments}

The authors thank CNPq, CAPES, and FAPEMIG for supporting this study.

\section{References}

Aymerich T, Martín B, Garriga M, Hugas M (2003) Microbial Quality and Direct PCR Identification of lactic acid bacteria and nonpathogenic staphylococci from artisanal low-acid sausages. Appl Environ Microbiol 69:4583-4594.

Beimfohr C, Ludwig W, Schleifer KH (1997) Rapid genotypic differentiation of Lactococcus lactis subspecies and biovar. Syst Appl Microbiol 20:216-221.

Chen H, Hoover DG (2003) Bacteriocins and their food applications. Compr Rev Food Sci 2:92-100.

Deegan LC, Cotter PD, Hill C, Ross PR (2006) Bacteriocins: Biological tools for bio-preservation and shelf-life extension. Int Dairy J 16:1058-1071.

Dutka-Malen S, Evers S, Courvalin P (1995) Detection of glycopeptide resistance genotypes and identification to the species level of clinically relevant enterococci by PCR. J Clin Microbiol 33:24-27.

Gomes BC, Esteves CT, Palazzo ICV, Darini ALC, Felis GE, Sechi LA, Franco BDGM, de Martinis ECP (2008) Prevalence and characterization of Enterococcus spp. isolated from Brazilian foods. Food Microbiol 25:668-675.

Mohania D, Nagpal R, Kumar M, Bhardwaj A, Yadav M, Jain S, Marotta F, Singh V, Parkash O, Yadav H (2008) Molecular approaches for identification and characterization of lactic acid bacteria. J Dig Dis 9:190-198.

Nishitani Y, Sasaki E, Fujisawa T, Osawa R (2004) Genotypic analyses of lactobacilli with a range of tannase activities isolated from human feces and fermented foods. Syst Appl Microbiol 27:109-117.

Ortolani MBT, Yamazi AK, Moraes PM, Viçosa GN, Nero LA (2010) Microbiological quality and safety ofraw milk and soft cheese and detection of autochthonous lactic acid bacteria with antagonistic activity against Listeria monocytogenes, Salmonella spp., and Staphylococcus aureus. Foodborne Pathog Dis 7:175-180.

Riley MA, Wertz JE (2002) Bacteriocins: evolution, ecology, and application. Annu Rev Microbiol 56:117-137.

Sterr Y, Weiss A, Schmidt H (2009) Evaluation of lactic acid bacteria for sourdough fermentation of amaranth. Int J Food Microbiol 136:75-82.

Velasco D, Perez S, Penã F, Dominguez MA, Cartelle M, Molina F, Moure R, Villanueva R, Bou G (2004) Lack of correlation between phenotypic techniques and PCR-based genotypic methods for identification of Enterococcus spp. Diagn Microbiol Infect Dis 49:151-156.

All the content of the journal, except where otherwise noted, is licensed under a Creative Commons License CC BY-NC. 